\title{
Quaderni
}

QUADERNI Communication, technologies, pouvoir

70 | Automne 2009

Think tanks, experts et pouvoirs

\section{Les principaux think tanks français}

\section{Geoffrey Geuens}

\section{(2) OpenEdition}

\section{Journals}

Édition électronique

URL : http://journals.openedition.org/quaderni/511

DOI : 10.4000/quaderni.511

ISSN : 2105-2956

\section{Éditeur}

Les éditions de la Maison des sciences de l'Homme

Édition imprimée

Date de publication : 5 octobre 2009

Pagination : 79-88

Référence électronique

Geoffrey Geuens, "Les principaux think tanks français », Quaderni [En ligne], 70 | Automne 2009, mis en ligne le 05 octobre 2011, consulté le 10 décembre 2020. URL : http://journals.openedition.org/ quaderni/511 ; DOI : https://doi.org/10.4000/quaderni.511 


\section{$D$ ossier}

\section{les principaux $\begin{aligned} & \text { INSTITUT FRANGAS } \\ & \text { DES RELATIONS INTERNATIONALES }\end{aligned}$ think tanks français ${ }^{1}$ \\ (IFRI) \\ Voir l'article de François Vergniolle de Chantal dans ce numéro.}

Geoffrey Geuens

Chargé de cours département des Arts et Sciences de la Communication Université de Liège

\section{INSTITUT DE RELATIONS \\ INTERNATIONALES \\ ET STRATÉGIQUES}

(IRIS)

\section{Historique}

L'Institut de relations internationales et stratégiques (IRIS) a été fondé en 1990 par Pascal Boniface. Diplômé de l'IEP de Paris et docteur en Droit international public, cet ancien conseiller du groupe parlementaire socialiste à l'Assemblée nationale était, au moment de la création de l'IRIS, conseiller des ministres de la Défense (Jean-Pierre Chevènement) et de l'Intérieur (Pierre Joxe). Ancien membre $\mathrm{du}$ Conseil consultatif pour les questions de désarmement de l'ONU (2001-2005), administrateur à l'Institut des Hautes études de la Défense nationale (1998-2004) et membre du Haut Conseil de la coopération internationale (1999-2003), Pascal Boniface démissionna du Parti socialiste en 2003, et cela suite à un différend portant sur la perception du conflit israélo-palestinien.

Dirigeants, chercheurs, adhérents

L'IRIS compte une trentaine de chercheurs (directeurs de recherche, chercheurs-enseignants, chercheurs associés) et dispose de deux présidents d'honneur en la personne de Pascal Lamy, directeur général de l'OMC, et Arthur Paecht, 
ancien vice-président de l'Assemblée nationale. Présidé par Jacques Boyon, ex-secrétaire d'Etat à la Défense, le conseil d'administration réunit Pascal Boniface, Renaud Donnedieu de Vabres, Hubert Védrine, François Scheer (Areva), Michel Barnier, Alain Bauer (AB Associates), Paul Boury (Boury et associés), Stéphane Fouks (EURORSCG), Marc-Antoine Jamet (LVMH), Bariza Khiari (sénatrice de Paris), Alain Marsaud, Yazid Sabeg (C\&S), Philippe Seguin, Lilian Thuram (Haut Conseil à l'Intégration), Manuel Valls, Denis Verret (EADS), le journaliste/producteur Bertrand de la Villardière et Francis Wurtz, président du groupe parlementaire européen Gauche unitaire européenne/Gauche verte nordique.

\section{Financement, partenariat(s)}

Modèle de coopération "public/privé », l'IRIS dispose essentiellement de trois sources de financement distinctes: un tiers des rentrées provient des contrats passés avec le ministère français de la Défense, un autre tiers de la formation offerte par l'Institut à sa centaine d'étudiants, un dernier tiers venant des cotisations d'entreprises privées et des contrats passés avec des organismes internationaux.

\section{Objectif(s)}

L'IRIS vise un triple objectif : celui tout d'abord de contribuer à la recherche, à la réflexion et au débat sur les questions stratégiques; celui de développer ensuite un centre d'expertise réellement indépendant et en prise constante sur l'actualité ; celui de créer enfin un espace de dialogue entre l'ensemble des composantes de ce qu'il est convenu d'appeler la « communauté stratégique». Experts venus de différents horizons professionnels et philosophiques, les responsables politiques, hauts fonctionnaires, industriels, militaires et autres universitaires associés à l'IRIS s'attachent par conséquent à mettre leur expertise au service de la pédagogie et, par là même, à vulgariser l'actualité internationale auprès de l'opinion publique.

\section{Activités}

Si il a récemment mis sur pied l'Institut privé de relations internationales et stratégiques (IPRIS) - établissement d'enseignement supérieur délivrant divers diplômes - l'IRIS est surtout connu pour deux publications de référence dans le domaine des relations internationales : la Revue internationale et stratégique (trimestrielle) et L'Année stratégique (un numéro par an). L'IRIS réalise en outre, pour le compte d'entreprises et d'organismes publics, des notes stratégiques et des études approfondies considérées par les professionnels comme de précieux outils d'aide à la décision.

\section{INSTITUT ASPEN FRANCE}

\section{Historique}

L'histoire de l'Aspen Institute - « maison mère » de l'Institut Aspen France - débute en 1949 lorsque l'industriel américain Walter Paepcke (Container Corporation of America), beau-frère du directeur du département d'État Paul Nitze, décide de réunir de prestigieuses personnalités du monde des affaires, de la politique et de la culture à la célébration du $200^{\mathrm{e}}$ anniversaire de la naissance de Goethe. Près de deux mille invités triés sur le volet (Albert Schweitzer, Thomas Mann, Arthur Rubinstein, etc.) se retrouvent ainsi au beau milieu des montagnes d'Aspen, dans 
le Colorado, pour une entrevue de vingt jours, accompagnés des plus grands noms de la presse internationale de l'époque. Si cet organisme, créé officiellement en 1950 sous le nom d'Aspen Institute for Humanistic Studies, était donc plutôt à l'origine un lieu de rencontres dédié aux discussions strictement intellectuelles et artistiques, il pris assez rapidement les contours d'un véritable think tank se consacrant dorénavant proritairement aux questions liées à la sécurité internationale, l'économie mondiale ou encore la lutte contre l'adversaire soviétique. Plus tard, soucieux de renforcer les relations de collaboration « trilatérales », les dirigeants de l'Aspen Institute favorisèrent l'éclosion de satellites en Allemagne (Berlin, 1974), en France (Paris, 1983) ${ }^{2}$, en Italie (Rome, 1984), au Japon (Tokyo, 1998 ) et plus récemment en Inde (New Delhi, 2004) et en Roumanie (Bucarest, 2006).

\section{Dirigeants, chercheurs, adhérents}

Officiellement indépendant de l'Aspen Institute, l'Institut Aspen France - comme ses homologues européens et asiatiques - entreprend des programmes de recherche et organise des cycles de conférences en toute autonomie. Il dispose également de ses propres instances dirigeantes : directoire, conseil de surveillance et conseil d'orientation. L'organisation en réseaux de cette galaxie de think tanks est notamment garantie par la présence d'une poignée de représentants des Aspen « affiliés » au sein des instances dirigeantes de chaque institut Aspen. Michel Pébéreau (BNP Paribas), président du conseil de surveillance de l'Institut Aspen France, siège ainsi au conseil d'administration de l'Aspen Institute de Washington, aux côtés de Madeleine Albright, Giulio Tremonti, de la reine Noor de Jordanie ou encore de Yotaro Kobayashi (Fuji Xerox).

Le directoire de l'Institut Aspen France est présidé par Elisabeth Lulin, une ancienne conseillère du ministre des Affaires étrangères Alain Juppé (1994-1995) devenue administratrice de la Société générale. Le conseil de surveillance, lequel définit la stratégie, fixe le cadre et la cohérence des activités de l'Institut, rassemble Michel Pébereau - déjà président de l'Institut de l'Entreprise - Jean-Paul Bailly (La Poste), Charles Beigbeder (Poweo), Pascal Cagni (Apple Europe), Philippe Cayla (Euronews), Pierre-André de Chalendar (Saint-Gobain), Jacques de Chilly (Aderly France), Thierry Desmarest (Total), Nicolas Dufourcq (Cap Gemini), Alain Grange Cabane (Fédération des Industries de la Parfumerie), Raphaël Hadas-Lebel (Conseil d'Orientation des Retraites), Jérôme Huret (consultant), Pierre Hurstel (Ernst \& Young), Neil Janin (McKinsey France), Hubert Joly (Carlson Wagon Lit France), Thierry de La Tour d'Artaise (Groupe SEB), Jacques Le Mercier (Fondation Banque Rhône-Alpes), Marwan Lahoud (MBDA), MarieChristine Levet (T-Online France), Olivier Mellerio (Interfinexa), Alain Mérieux (BioMérieux), Gérard Mestrallet (GDF Suez), Nicolas Naudin (EADS), Gilles Pelisson (Accor), Arnaud de Puyfontaine (Mondadori France), Bertrand Richard (SpencerStuart France), Augustin de Romanet de Beaune (CDC), Patrice Vial (Hawkpoint Partners) et Remy Weber (Lyonnaise de Banque).

Quant au conseil d'orientation de l'Institut, il compte notamment au rang de ses membres Raymond Barre, Jacques Delors, Jean-Claude Trichet, Michel Barnier, Jacques Barrot, Nicole Notat, le Frère Samuel ou encore Hubert Védrine. 


\section{Financement, partenariat(s)}

Les principaux partenaires de l'institut sont des collectivités territoriales (Ville de Lyon, Grand Lyon, Région Rhône-Alpes, CCI de Lyon, Département du Rhône, Ville de Grenoble, Ville de Saint-Etienne) et de très nombreuses grandes entreprises françaises et étrangères (Air liquide, Adecco, BioMérieux, BNP, Bayer, Bouygues, CDC, France Télécom, GDF, Renault, Veolia, etc.). Outre l'Aspen Institute de Washington et ses «antennes », un certain nombre de think tanks collaborent intensivement avec l'Institut Aspen France: Bruegel, European Policy Center (EPC), Fondation Robert Schuman, Fondation Gabriel Péri, Fondation Jean Jaurès, Fondation pour l'Innovation Politique, Friedrich Ebert Stiftung, The German Marshall Fund of the United States, The Japan Institute of International Affairs (JIIA).

\section{Objectif(s)}

Officiellement, l'Institut ne mène pas d'action de lobbying, ne fait pas de recommandation ou de proposition en son nom propre, et ne développe pas non plus d'activité spécifique de conseil aux entreprises et partenaires. Aspen France s'est en réalité fixé un seul et même objectif : celui de demeurer un forum international d'échange et de réflexion de tout premier plan pour des décideurs de haut niveau issus d'univers culturels et professionnels distincts. Dirigeants d'entreprises, responsables politiques et cadres supérieurs des organisations internationales (Banque mondiale, OMC, FMI, OCDE...) y côtoient ainsi universitaires, mandataires syndicaux et autres journalistes de renom, désireux d'approfondir la réflexion sur ces thèmes de travail privilégiés par l'Institut que sont les relations transatlantiques, l'Afrique, la Méditerranée, les religions ou encore la question du développement territorial.

\section{Activités}

Lieu de réflexions et d'échanges avant toute autre chose, l'Institut Aspen France organise régulièrement des déjeuners, dîners ou débats autour d'une thématique spécifique et d'un prestigieux invité. Il convient de noter que les participants à ces dialogues informels interviennent systématiquement à titre personnel; la confidentialité, la spontanéité et l'efficacité de ces rencontres étant assurées par les organisateurs de l'événement. Si les journalistes, invités comme observateurs en certaines occasions, se voient ainsi interdire le droit de reproduire publiquement le contenu des discussions, des notes de synthèse sont disponibles sur le site internet de l'Institut.

\section{INSTITUT MONTAIGNE}

\section{Historique}

L'Institut Montaigne a été créé en 2000 par Claude Bébéar, lequel venait alors de quitter la présidence du directoire d'AXA pour prendre la tête de son conseil de surveillance. Libéré de ses fonctions exécutives, le «parrain » de l'économie française - comme aiment à le présenter les journalistes financiers - s'associe alors à deux autres grands noms du monde des affaires : Henri Lachmann (Schneider Electric), administrateur d'AXA et intime du président Chirac, et Alain Mérieux, PDG du groupe pharmaceutique éponyme et ancien premier vice-président du Conseil Régional Rhône- 
Alpes aux côtés de Charles Millon. Quant à Bernard de La Rochefoucauld, fondateur de l'Institut La Boétie, il apporta à l'Institut Montaigne son fonds documentaire et lui inspira un nom rappelant, à son tour, les beaux jours de La Pléiade. Signalons enfin que Claude Bébéar a également été à l'origine d'Entreprises et Cité, un club de réflexion créé en 1983 dans la périphérie du Parti républicain et de l'UDF dont Bébéar fut membre lorsqu'il occupa le poste de Premier adjoint au Maire de Rouen (1989). Regroupant alors vingt-huit PDG, ce cercle mondain comptait déjà au rang de ses membres Henri Lachmann.

\section{Dirigeants, chercheurs, adhérents}

Rassemblant une septantaine de membres - parmi lesquels les dirigeants de la plupart des sociétés du CAC40 - l'Institut Montaigne s'est doté d'un comité directeur dirigé par Claude Bébéar (président), Bernard de La Rochefoucauld (président d'honneur), Henri Lachmann (vice-président) et François Rachline (directeur général), professeur à Sciences Po. Quant aux autres membres de ce comité directeur, ils se nomment Nicolas Baverez, Jacques Bentz (Tecnet Participations), Guy Carcassonne, Christian Forestier (Haut Conseil d'Evaluation de l'Ecole), Françoise Holder (Holder SAS), Ezra Suleiman (Princeton University, AXA), Philippe Wahl (Groupe Bolloré), l'avocat d'affaires Jean-Paul Tran Thiet, Lionel Zinzou (PAI Partners) et Ana Palacio (Banque mondiale). Le conseil d'orientation, quant à lui, rassemble notamment Olivier Blanchard (MIT, Warburg Pincus), Jean-Pierre Boisivon (Institut de 1'Entreprise), François Ewald, Michel Godet (CNAM), Erik Izraelewicz (La Tribune),
Elisabeth Lulin (Institut Aspen France) et AlainGérard Slama (Le Figaro).

\section{Financement, partenariat(s)}

Refusant toute subvention publique, l'Institut Montaigne est financé par plus de 90 petites et grandes entreprises - chacune d'elles représentant moins de $2 \%$ du budget total (3,5 millions d'euros) - ainsi que par les dons de quelque 200 généreux donateurs. De nombreux évènements sont par ailleurs organisés en partenariat avec des médias (The Economist), des think tanks (Center for European Reform, Konrad Adenauer Stiftung, Notre Europe, Institut Aspen France) ou encore des organisations internationales (OCDE).

\section{Objectif(s)}

Comme toute boîte à idées, l'Institut Montaigne a deux objectifs principaux : influencer le débat public en apportant des idées «pragmatiques » et «innovantes », et participer à la définition des politiques publiques. Organisant l'essentiel des activités du think tank autour de trois grands axes de recherche - mobilité/cohésion sociale, modernisation de la sphère publique, stratégie économique européenne - les dirigeants de l'Institut se refusent à prendre ouvertement position dans le débat politique et électoral tout en se réclamant d'un libéralisme économique seul à même de «débloquer» la France. Travaillant dans l'intérêt général et soucieux de pragmatisme, à suivre ses responsables, l'Institut Montaigne est, à mieux y regarder, une boîte à idées « néolibérale » singulièrement proche des milieux économiques français.

\section{Activités}

Les chercheurs et dirigeants de l'Institut 
Montaigne proposent des recommandations faisant l'objet d'un « lobbying actif auprès des décideurs publics », ce qui suppose la mise en place, l'extension continue et la consolidation d'un réseau de relations serré avec les pouvoirs publics et l'administration en place. L'influence - toujours difficilement mesurable - de ce think tank s'exerce notamment à travers ses conférences : les débats «Libres Échanges », organisés une fois par mois à l'auditorium AXA en partenariat avec « Les Mardis de l'ESSEC », réunissent trois à quatre intervenants et jusqu'à 400 participants; quant au séminaire de réflexion annuel consacré à la problématique des «think tanks », il est organisé au Conseil économique et social. Côté publications, trois types de documents sont téléchargeables et mis à la disposition du grand public sur le site Internet de 1'Institut: les «rapports » rassemblent les conclusions émanant des groupes de travail thématiques, les «notes» permettent à un chercheur de présenter sa vision des questions faisant débat, l'«Amicus Curiae», enfin, constitue en quelque sorte une réaction à chaud de l'Institut devant l'actualité immédiate.

\section{FONDATION POUR L'INNOVATION POLITIQUE}

\section{Historique}

La Fondation pour l'Innovation politique (FONDAPOL) a été créée en janvier 2004 par l'UMP. C'est Jérôme Monod, alors conseiller de Jacques Chirac à l'Elysée et président honoraire du groupe Suez, qui prit rapidement les commandes de ce think tank reconnu, deux mois plus tard seulement, établissement d'utilité publique par le Premier ministre Jean-Pierre Raffarin.

\section{Dirigeants, chercheurs, adhérents}

Les structures dirigeantes de cette boîte à idées rassemblent un grand nombre de responsables publics, d'hommes d'affaires, d'intellectuels et de journalistes hexagonaux, européens ou extracontinentaux recrutés - pour la très grande majorité d'entre eux - au sein de cette vaste famille politique qu'est la droite française. Présidé par Nicolas Bazire (Groupe Arnault), conseiller personnel du Président Sarkozy et ancien directeur de cabinet du Premier ministre Balladur, le conseil de surveillance se compose du vice-président Charles Beigbeder (Poweo), du président honoraire Jérôme Monod, de Cheick Modibo Diarra (Microsoft Afrique), Cyrille Bardon (Cabinet Bardon-de Faÿ), Sir Stuart Bell (député travailliste), Barid Baran Bhattacharya (économiste indien), Aldo Cardoso (GDF-Suez), Grégoire Chertok (Rothschild \& Cie), Laurent Cohen-Tanugi, Geneviève Ferone (Veolia), Pierre Giacometti (Ipsos), Jiang Changjian (Université de Fudan), Francis Mer (Safran), Jean-Claude Paye et Monique Sassier, inspectrice générale de l'Administration de l'Éducation nationale et de la Recherche. Présidé par François Ewald, le Conseil scientifique, quant à lui, se compose notamment de Stéphane Courtois, Patrick Lozès (Conseil représentatif des Associations noires de France), Pascal Perrineau (CEVIPOF) et AlainGérard Slama (Institut Montaigne). Signalons enfin que le directeur général de FONDAPOL est Dominique Reynié, professeur des universités à Sciences Po, et que d'autres grands noms de la vie politique, médiatique et intellectuelle française conseillent la Fondation que l'on songe à Alexandre Adler, Alain Besançon, Raymond Boudon, Marcel Gauchet, Emmanuel Le Roy Ladurie, Gilles Kepel, Elisabeth Lulin (Institut 
Montaigne, Aspen France), Michel Maffesoli, Pierre Nora ou encore Pierre-André Taguieff.

\section{Financement, partenariat(s)}

FONDAPOL est essentiellement financée par les pouvoirs publics et de généreux donateurs, qu'il s'agisse de personnes physiques, de diverses institutions ou d'entreprises partenaires autorisées à « formuler des recommandations au sein de la convention » dès que leur versement annuel dépasse la somme des 30000 euros. Refusant aujourd'hui tout financement direct émanant d'un quelconque parti politique, FONDAPOL reçoit cependant - comme toute « fondation démocratiques à vocation politique » - une dotation des services du Premier ministre. Ce think tank bénéficie qui plus est, dans l'organisation de certains évènements, de la collaboration de plusieurs prestigieuses boîtes à idées telles que la Fondation Konrad Adenauer (CDU), la Fondation Hanns-Seidel (CSU bavaroise), la FAES (Parti populaire espagnol), le Carnegie Endowment for International Peace, Brookings Institution ou encore les groupements néo-conservateurs américains Heritage Foundation et Hudson Institute.

\section{Objectif(s)}

Si la mission de FONDAPOL fut, à ses origines, de participer à la refondation doctrinale de l'UMP - lequel parti contribua un temps jusqu'à $17 \%$ de son budget - ce think tank s'est progressivement émancipé de cette tutelle pour se recentrer sur les problématiques du vieillissement démographique, de l'évolution climatique, de la globalisation des sphères marchande et financière, des pays en voie de développement ou encore de l'économie numérique comme moteur de croissance.
Défenseur de « la liberté et de l'initiative individuelle », FONDAPOL continue aujourd'hui de se réclamer de la « famille politique de la droite et du centre ».

\section{Activités}

2050 est la revue trimestrielle publiée par FONDAPOL et dirigée par Jean de Boishue, ancien secrétaire d'État à l'Enseignement supérieur (1995) et actuel chargé de mission auprès du Premier ministre François Fillon. Ces dernières années, FONDAPOL s'est notamment fait remarquer pour la création d'une Université Numérique Francophone Mondiale (UNFM) destinée à assurer le développement du téléenseignement dans les pays francophones du sud, ce centre de réflexion et d'action a été cofondé avec le PDG de Microsoft Afrique Cheick Modibo Diarra. L'UNFM dispose notamment de sites de référence au Mali (Bamako), au Burkina Faso (Ouagadougou) et en République du Congo (Brazzaville).

\section{EN TEMPS RÉEL}

\section{Historique}

En Temps Réel est une association généraliste de débats et de réflexion créée en 2000, peu après l'autodissolution de la Fondation SaintSimon. Les dirigeants historiques de ce fer de lance du social-libéralisme à la française ayant refusé aux jeunes «saint-simoniens » le droit de relancer la Fondation, ceux-ci mirent alors sur pied un nouveau groupe de réflexion intéressé par des questions globales telles que la construction européenne, la régulation de l'économie mondiale ou encore l'élaboration d'un projet politique «progressiste» pour le 
$\mathrm{XXI}^{\mathrm{e}}$ siècle, et cela au plan international.

\section{Dirigeants, chercheurs, adhérents}

Le bureau exécutif d'En Temps Réel se compose du président Gilles de Margerie (Crédit agricole), du trésorier Philippe Crouzet (Vallourec) et du secrétaire Bernard Spitz (BSConseil). Le conseil d'administration rassemble, de son côté, Stéphane Boujnah (Deutsche Bank), Nicolas Dufourcq (Cap Gemini), Laurent Joffrin (Libération), Olivier Nora (Editions Grasset), Denis Olivennes (Nouvel Observateur) et Emanuelle Wargon (directrice de cabinet de Martin Hirsch). Le conseil d'orientation rassemble enfin Laure Adler (ex-France Culture), Renaud Dehousse (Centre Européen de Sciences Po), Marcel Gauchet (Le Débat), François Heisbourg (Fondation pour la Recherche stratégique), Anne-Marie Idrac (secrétaire d'État au Commerce extérieur), Jean-Noël Jeanneney (ex-BNF), Pascal Lamy (OMC), Nicole Notat, Jean Pisani-Ferry (Paris Dauphine), Jean-François Rischard (ex-Banque mondiale), Richard Robert (Cadres CFDT), Nicolas Veron (think tank Bruegel), François Villeroy de Galhau (Cétélem) et Charles Wyplosz (Université de Genève, FONDAPOL).

\section{Financement, partenariat(s)}

Le financement d'En Temps Réel repose intégralement sur les cotisations de ses membres et entreprises-partenaires.

\section{Objectif(s)}

Mener une réflexion de fond sur des problématiques aussi diverses que l'avenir du réformisme en France, la question sociale ou encore la démocratisation du Moyen-Orient.
Activités

En Temps Réel organise des conférences-débats et des séminaires thématiques. Les travaux d'En Temps Réel sont publiés sous forme de cahiers envoyés à divers responsables politiques, économiques, académiques, médiatiques et syndicaux. L'association finance en outre les projets de recherche de jeunes doctorants.

\section{LA RÉPUBLIQUE DES IDÉES}

\section{Historique}

La République des Idées a été mise sur pied en 2002 à l'initiative de Pierre Rosanvallon, l'une des chevilles ouvrières de la défunte Fondation Saint-Simon dont certains membres avaient déjà, deux ans auparavant, participé au lancement d'En Temps Réel.

\section{Dirigeants, chercheurs, adhérents}

Les principaux responsables de La République des Idées se nomment Pierre Rosanvallon (président), Olivier Mongin (vice-président, Esprit,) et Jean Peyrelevade (trésorier), tous trois anciens « saint-simoniens ».

\section{Financement, partenariat(s)}

La vente des livres publiés en collaboration avec les éditions du Seuil constitue près de la moitié des recettes du think tank, le restant provenant des cotisations d'entreprises et des contrats de recherche publics ou privés.

\section{Objectif(s)}

Transformer les sciences humaines et mettre à la disposition de l'opinion publique et des chercheurs de nouveaux outils de réflexion, d'analyse et de compréhension du monde, tels 
sont les principaux objectifs que s'est donnés La République des Idées. Cet ambitieux projet intellectuel constitue en quelque sorte la colonne vertébrale d'un ensemble de réflexions et de recherches se distribuant selon quatre grands axes de travail: les transformations du capitalisme; la démocratie européenne, l'élargissement à l'Est, la mise à l'épreuve de la relation transatlantique, les relations à l'Islam et aux mondes émergents; la géopolitique de la mondialisation; les relations nouvelles entre individualisme et solidarités sociales.

\section{Activités}

La République des Idées ne pratique pas le lobbying et refuse a priori tout travail d'expertise opérationnelle pour les décideurs, publics comme privés. Sa politique volontaire de production intellectuelle s'exprime ainsi à travers trois principaux canaux : la revue $L a$ vie des idées, les conférences, colloques et débats qu'elle organise, et surtout sa collection d'essais au Seuil au catalogue duquel on retrouve Christian Baudelot et Roger Establet (L'élitisme républicain), Jean Peyrelevade (Le capitalisme total), Pascal Lamy (La démocratie-monde) ou encore Robert Castel (L'insécurité sociale).

\section{INSTITUT DE L'ENTREPRISE}

\section{Historique}

Créé en 1975, l'Institut de l'entreprise voulait doter les entreprises «d'un organe de réflexion, indépendant de toutmandatsyndicaloupolitique, qui leur permette d'affiner leur approche des questions économiques, sociales et sociétales ». Ses fondateurs, François Ceyrac (CNPF), Jean
Chenevier (CNPF/CRC) et François Dalle (L'Oréal), souhaitaient par là même prolonger l'action du Centre de recherches et d'études des chefs d'entreprise (CRC), créé en 1953 par Georges Villiers.

\section{Dirigeants, chercheurs, adhérents}

L'Institut de l'entreprise réunit aujourd'hui plus de 120 adhérents, dont les activités couvrent l'ensemble des secteurs économiques, générant un chiffre d'affaires cumulé qui représente plus de $20 \%$ du PIB marchand de la France. À la fois différent des organisations professionnelles et en contact permanent avec elles, l'Institut se distingue par sa capacité à échapper aux pressions de la conjoncture immédiate. Plus des deux tiers des entreprises cotées au CAC 40 sont membres de l'Institut. L'Institut est piloté par un conseil d'orientation composé d'une quinzaine de PDG fixant la politique de l'Institut, déterminant les objectifs à moyen et long termes, et interrogeant les moyens nécessaires à leur réalisation. Présidé par Michel Pébéreau (BNP Paribas), le conseil d'orientation se compose de Robert Baconnier (ANSA), Pierre Bellon (Sodexho), Philippe Carli (Siemens France), Françoise Gri (Manpower France), Xavier Huillard (Vinci), Henri Lachmann (Schneider Electric), Gérard Mestrallet (GDF Suez), Henri Proglio (Veolia), Pierre Richard (Dexia), Frédéric Saint-Geours (UIMM), Ernest-Antoine Seillière (Wendel), Pierre Simon (CCI de Paris), Jean-François Théodore (NYSE Euronext), Jean-Philippe Thierry (AGF) et Pierre-Sébastien Thill (CMS Bureau Francis Lefebvre).

\section{Financement, partenariat(s)}

L'Institut de l'entreprise est uniquement 
financé par les cotisations de ses membres tels que Accor, AGF, Air Liquide, BNP Paribas, Carrefour, EDF, France Telecom, HEC, ESSEC, KPMG, Lafarge, PSA, Publicis Consultants, Safran, Suez-GDF ou encore Total. On compte parmi les partenaires internationaux de l'Institut, un certain nombre d'organisations étrangères situées dans la périphérie directe d'organisations patronales nationales telles que le Committee for Economic Development (États-Unis), l'Institut der deutschen Wirtschaft (Allemagne) ou encore le Keizai Doyukai (Japon).

\section{Objectif(s)}

L'Institut de l'Entreprise se présente volontiers comme un lieu d'élaboration d'une "pensée managériale moderne » et un centre de réflexion sociétal. Les objectifs que s'est donnés l'Institut de l'Entreprise en font une institution singulière située au croisement des groupes de réflexion privés, des organisations patronales traditionnelles et des sociétés de relations publiques.

\section{Activités}

Les diverses activités organisées par l'Institut de l'Entreprise se structurent autour de trois pôles distincts : le "pôle de réflexion" chapeaute diverses commissions d'étude prospective réunissant chefs d'entreprises et experts, et aboutissant à la rédaction de rapports largement diffusés par la suite ; le pôle « lieu de rencontre » rassemble des dirigeants de sociétés, responsables politiques et représentant du monde académique à l'occasion de déjeuners informels, colloques et autres séminaires internationaux; quant au «pôle de formation », il s'attache à travers des stages d'immersion ou encore des conférences- débats à sensibiliser le grand public aux réalités concrètes du monde de l'entreprise. Ce pôle de formation s'adresse aujourd'hui à trois publics types: les «jeunes élites», issues d'horizons variés et appelées à exercer des postes à responsabilité dans le futur, les professeurs de Sciences Économiques et Sociales des lycées, les élèves journalistes. Signalons enfin que l'Institut de l'entreprise contrôle actuellement via sa filiale Société d'études, de recherche et de publications économiques (SERPE) la revue trimestrielle Sociétal, une revue de prospective née en 1996 du rapprochement de deux publications éditées par Bertrand de Jouvenel (Futuribles).

1. Les informations rassemblées dans la présente annexe sont tirées des portails Internet officiels des think tanks répertoriés et des recherches entreprises, depuis plusieurs années maintenant, par l'auteur.

2. L'Institut Aspen France s'est établi à Lyon en 1994 sous l'impulsion de Raymond Barre, président du think tank et futur maire de Lyon. 